\title{
Sacrifices de rennes et pétrole : dialogue entre rituel et droits des peuples autochtones
}

\section{Art Leete}

Traducteur : Eva Toulouze

\section{(2)enEdition \\ Journals}

Édition électronique

URL : https://journals.openedition.org/efo/4117

DOI : $10.4000 /$ efo. 4117

ISSN : 2275-1947

Cet article est une traduction de :

Põhjapõdraohverdus ja nafta: põlisrahvaste õiguse ja rituaali dialoog - URL : https:// journals.openedition.org/efo/4760 [et]

\section{Éditeur}

INALCO

\section{Édition imprimée}

Date de publication : 1 janvier 2014

ISBN : 978-2-343-05394-3

ISSN : 0071-2051

\section{Référence électronique}

Art Leete, "Sacrifices de rennes et pétrole : dialogue entre rituel et droits des peuples autochtones », Études finno-ougriennes [En ligne], 46 | 2014, mis en ligne le 14 octobre 2015, consulté le 06 octobre 2022. URL : http://journals.openedition.org/efo/4117 ; DOI : https://doi.org/10.4000/efo.4117

Ce document a été généré automatiquement le 6 octobre 2022.

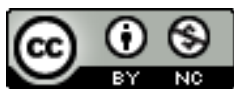

Creative Commons - Attribution - Pas d'Utilisation Commerciale 4.0 International - CC BY-NC 4.0 https://creativecommons.org/licenses/by-nc/4.0/ 


\title{
Sacrifices de rennes et pétrole : dialogue entre rituel et droits des peuples autochtones
}

\author{
Art Leete
}

Traduction : Eva Toulouze

\section{Introduction}

1 Les habitants autochtones de la Sibérie occidentale sont les Ougriens de l'Ob' (Khantys et Mansis) et les Samoyèdes (Nenetses de la toundra et des forêts). Cette même région a été, au cours de ces cinquante dernières années, l'aire d'exploitation industrielle du gaz et du pétrole la plus importante pour la Russie. Les principales activités de subsistance des autochtones (la chasse, la pêche et l'élevage du renne) sont toutes en rapport étroit avec l'environnement naturel, de sorte que la production de gaz et de pétrole limite de plus en plus leurs possibilités de vivre suivant le mode de vie traditionnel, aussi bien en raison de la pression exercée sur l'environnement que du fait de la transformation dramatique de la structure et des effectifs de la population (depuis les années 1960, le nombre des habitants de la région a été multiplié par quinze alors que le nombre d'autochtones est resté relativement stable). Ces évolutions aboutissent au recul du mode de vie traditionnel; de plus, elles conduisent à une réévaluation nouvelle et positive, à une actualisation puissante de la vision du monde traditionnelle et des pratiques rituelles.

2 À l'époque postsoviétique, les autochtones de Sibérie occidentale ont organisé des sacrifices collectifs de rennes, plus nombreux et plus publics qu'avant, ainsi que d'autres initiatives où les rituels traditionnels sont liés aux messages et aux besoins de leurs élites politiques. Ces rituels, dont la fonction a été ainsi partiellement transformée, reflètent à leur manière les évolutions sociales et économiques de la région. 
Plusieurs auteurs se sont penchés sur les rituels collectifs à revendications politiques, parfois tournés vers l'opinion publique, des autochtones de Sibérie occidentale. Les recherches d'Anzori Barkalaja $(1997$; 1999) se concentrent sur les sacrifices de rennes collectifs des Khantys du Pim, lesquels avaient pour but de vénérer les esprits protecteurs de la région et d'élever le sens d'appartenance à la communauté locale. Pour ma part, j'ai analysé un sacrifice khanty mis en scène par l'administration à l'intention d'une délégation internationale sur le Tromagan (Leete 2004). Cette recherche est particulièrement proche de celles d'Eva Toulouze et de Liivo Niglas sur la problématique des sacrifices de rennes chez les Nenetses de la toundra et de la forêt (Niglas 1997; Niglas, Toulouze 2004; Toulouze, Niglas 2012). Différents types de sacrifices collectifs khantys ont été analysés comparativement par exemple par Kustaa Frederik Karjalainen (1918), Andrew Wiget et Olga Balalaeva (2011), mais aussi par Marjorie Mandelstam Balzer (1999). Natalja Novikova a abordé à plusieurs reprises la problématique du «renne du président» $(1998 ; 1999 a ; 2000 ; 2002)$, qui est en lien direct avec la cérémonie traitée dans le présent article.

4 Les analyses réalisées jusqu'ici se sont concentrées sur tel ou tel rituel, sur l'étude d'éléments de ce rituel ou, plus généralement, sur l'interprétation du rôle du renne (aussi bien économique, rituel que politique). Dans cette étude, je vais essayer d'intégrer toutes ces dimensions. J'étudierai la manière dont les éléments des rituels peuvent être interprétés un par un, comment ils forment un tout, un ensemble sacral traditionnel, et comment le rituel est mis en relation avec le combat actuel contre l'industrie du pétrole et les administrateurs politiques.

5 J'analyserai la situation actuelle des peuples autochtones de Sibérie occidentale, et le rôle potentiel du rituel pour refléter la situation politique d'ensemble et agir sur elle à travers les événements liés au sacrifice collectif de rennes du 19 avril 1997 sur le site sacré au bord du lac Num-to dans l'okroug autonome de Hanty-mansijsk / Jugra, raïon de Belojarsk. L'objectif principal de cette cérémonie était d'empêcher l'exploitation du pétrole prévu par les compagnies pétrolières et partiellement commencé à proximité du lac Num-to. En même temps, dans cet article, le problème de l'industrie pétrolière dans ses rapports avec les autochtones reste secondaire. Je me concentrerai sur l'analyse aussi dense que possible du rituel et je ne renverrai à l'industrie pétrolière qu'accessoirement. Le lien entre industrie pétrolière et autochtones mérite une discussion plus approfondie et une analyse ad hoc.

6 Pour aborder ce rituel, je suis les principes dits de la description dense (cf. Geertz 2001 [1973]). J'ai déjà abordé cette cérémonie dans mes écrits (Leete 1997; 1999; Leete, Niglas, Barkalaja 2001), mais cette analyse-ci est bien plus approfondie que les précédentes. Je publie le texte des interventions télévisées effectuées au cours du rituel, sachant que le porte-parole des autochtones, Juri Vella (Ajvaseda) (1948-2013) était intéressé à ce que ces matériaux passent dans l'une des principales chaines nationales de Russie. Le leader des autochtones souhaitait faire connaitre largement les problèmes liés aux droits des autochtones et à la préservation de l'environnement, ainsi que les problèmes culturels et écologiques dus à l'extension de l'industrie pétrolière. Juri Vella a également affirmé à plusieurs reprises que, pour lui, toute attention publique portée à la situation des autochtones de Sibérie occidentale était d'une importance primordiale.

7 Cela fait des décennies que la tradition de la recherche en anthropologie met l'accent sur la nécessité de « donner la parole » aux informateurs autochtones (cf. par exemple Radin 2008 [1956]). En même temps, il n'est pas facile de trouver la bonne mesure dans 
le mélange du texte des informateurs et des interprétations du chercheur: si l'on présente de longs textes avec peu de commentaires, l'explication du contexte peut être insuffisante et la compréhension du lecteur peut en souffrir. Si l'on se contente au contraire de renvoyer au discours autochtone par quelques allusions, le lecteur est contraint de faire entièrement confiance au chercheur sur l'adéquation des idées autochtones à la réalité. J'ai choisi une approche médiane: je donne largement la parole à notre principal informateur, tout en essayant de contextualiser mes explications grâce à la littérature précédemment existante et aux informations reçues d'autres interlocuteurs.

8 Je présente ces événements comme des exemples éloquents de la lutte autochtone actuelle pour la survie. L'affirmation de la dignité, des droits de l'homme, de l'environnement des autochtones passe en l'occurrence par le contexte rituel. Cette dimension permet aux autochtones de souligner l'originalité et la permanence de leur culture. Par ailleurs, leur leader politique et spirituel, Juri Vella, témoigne ici d'une sensibilité politique exceptionnelle, qui rend le conflit avec les compagnies pétrolières particulièrement curieux. C'est bien l'interpénétration d'un rituel authentique et de la lutte politique actuelle qui constitue le thème de cette recherche.

C'est par hasard que je me suis retrouvé à la cérémonie de Num-to - il était impossible de prévoir que cet événement serait le point de départ de la passionnante histoire du « renne du président ». Je faisais mon terrain à Hanty-Mansijsk et je me suis rendu en visite chez le folkloriste Timofej Moldanov. Tout en buvant le thé, il m'a demandé si je ne voudrais pas aller le lendemain à une cérémonie à Num-to. Sans rien savoir de ce qui allait se passer, je fus immédiatement d'accord. Je me disais que c'était la manière dont les choses se produisent en Sibérie : on se trouve comme par hasard à tel ou tel endroit, et l'on apprend quelque chose. J'avais constaté que c'était là une technique efficace de terrain : attendre, dans l'espoir que des choses se passent, sans pour autant presser mes interlocuteurs par mon insistance.

10 Le lendemain nous montâmes dans un hélicoptère (que l'un des organisateurs de la cérémonie, Juri Vella, avait obtenu, en échange de la promesse de ne pas organiser de manifestation hostile à l'industrie pétrolière ${ }^{1}$ ) et nous partîmes à Num-to. Avant d'y arriver, nous survolâmes presque toute la Sibérie occidentale, collectant dans les campements de la taïga les Khantys et les Nenetses qui se rendaient à la cérémonie, dont Juri Vella lui-même. La description que je présente ici s'appuie sur les notes que j'ai écrites après la cérémonie, le soir du 19 avril 1996 au village de Num-to, ainsi que sur les commentaires de certains participants.

11 Le sacrifice des rennes eut lieu sur un site sacré situé sur une petite île proche de la rive sud du lac Num-to, à peu près à $1,5 \mathrm{~km}$ à l'est du village. Sur la berge du lac, à environ une centaine de mètres, il y avait sept cèdres sacrés. L'île était couverte de bouleaux. $\mathrm{Au}$ moment de la cérémonie, le temps était froid et venteux, il faisait entre - 15 et $20^{\circ} \mathrm{C}$.

12 Lors du rituel, mon principal informateur, celui qui m'a expliqué ce qui se passait, était Stepan Randymov (1941-2005). Je m'appuie largement sur ses commentaires. Ceci tient au fait que ses explications sur le contexte général du rituel et sur bien des détails étaient systématiques, harmonieusement en phase avec le mode de pensée des autochtones et présentées de manière convaincante. Il est apparu clairement en parlant avec lui que Stepan prend le rituel au sérieux et tente de l'expliciter au mieux, le plus honnêtement possible. En suivant les activités, je constatai que c'était une 
personne particulière. La plupart des participants étaient tranquillement efficaces, voire relativement indifférents ou négligents. Stepan, lui, était tout le temps en activité et j'eus l'impression qu'il se concentrait sur des détails du rituel qui étaient passés inaperçus aux yeux des autres. Le soir, je lui rendis visite et je constatai que j'avais eu raison: Stepan n'avait pas une haute opinion de l'engagement des Nenetses et avait veillé discrètement à des détails négligés.

J'ai été également impressionné par son comportement de manière générale : sur la base de mon expérience, celui-ci se rapprochait du prototype de l'étiquette khantye. Ses activités quotidiennes (pour le peu que j'ai pu en observer) étaient parsemées d'éléments déterminés par sa religion. Par exemple quand je suis allé chez lui à Num-to, il a exigé qu'on me fasse un cadeau, car quand quelqu'un vient de loin, il faut lui donner quelque chose. C'était pour lui une question qui le touchait aussi au niveau des émotions : il fallait qu'il y ait de l'ordre dans les choses de ce monde.

\section{Les femmes et les lieux sacrés}

Le rituel n'a pas vu la participation de tous les habitants de la région qui auraient pu en principe y assister. Au moment du rituel, nous avons vu passer par le lieu du rituel, sur le lac, des traîneaux qui n'ont pas accordé à la cérémonie la moindre attention :

Personne n'est invité personnellement. Ceux qui veulent doivent venir eux-mêmes sur place. Ces deux-là allaient à leur village, à leur čum en passant par le lac. En général on informe tout le monde de la cérémonie. On en parle à tous ceux qui veulent y participer. Les jeunes ne veulent pas. Leurs parents n'en faisaient pas et eux n'en veulent pas.

Les participants à la cérémonie, hommes et femmes, se rendirent sur place en traversant le lac gelé dans des traîneaux tirés par des attelages de rennes. Trois rennes, le nombre nécessaire pour un attelage, furent attachés sur le lieu du sacrifice à un bouleau. Parmi les participants, il y avait aussi des femmes, venant aussi bien de Numto que de Hanty-Mansijsk; d'après Stepan, elles étaient autorisées à être présentes parce que ce sacrifice était dédié à tous les dieux en même temps. Si le destinataire avait été uniquement "le dieu suprême, le dieu blanc du ciel», seuls les hommes auraient été en droit d'y participer. Stepan commenta en détail les rôles et les droits différents des hommes et des femmes dans le contexte rituel :

Et si l'on ramène à la maison [la viande de renne qui reste après le sacrifice], on $\mathrm{n}$ '[en] donne pas à manger aux femmes. Ni congelée, ni cuite. Seuls les hommes [en mangent]. Mais chez les Nenetses il y a des familles comme ça. Ils font des sacrifices avec les femmes aux dieux du ciel, du soleil, à la lune, à tous les dieux ensemble. Il y a des Nenetses qui font ça, j'en connais. Alors c'est permis. Ils suspendaient à l'intention du Dieu blanc [à un bouleau] une peau [de renne] blanc avec la tête. Ils arrivaient pour la prière en compagnie des femmes. C'est autorisé. Ils rentraient chez eux, et les femmes ont tout aussi le droit de manger la viande. Les hommes aussi. Mais il y a aussi des lieux où ne vont que les hommes. Ce sont les lieux en hauteur. On monte sur une hauteur et on regarde... Oh! Au-dessus de la forêt, on aperçoit un lieu sacré. En fait, quand on se déplace en attelage de rennes, [parfois] il est à quarante ou cinquante kilomètres de distance. Ces lieux sont situés surtout sur les hauts cours des rivières, entre les cours d'eau. Dans cette région, au bord du Kazym et ailleurs, il y en a. Ce sont des endroits où les femmes ne mettent pas les pieds. 
Stepan reconnaît ainsi que les droits rituels des femmes peuvent varier suivant les familles. Il souligne également que les possibilités des femmes dans le cycle rituel sont plus étendues chez les Nenetses. Cela peut être une référence à la liberté sociale, plus large chez les Nenetses. En même temps on a l'impression que ce que Stepan veut dire, c'est qu'à ses yeux les Nenetses se comportent de manière erronée, laissant trop de place aux femmes. Toute l'explication par Stepan de la cérémonie est traversée par une exigence de respect très strict des règles rituelles. Comme il avait une vision très précise des détails de la cérémonie, il était en mesure de voir partout les erreurs des autres. À mon sens, cela n'était pas dû à une attitude malveillante vis à vis des Nenetses ou des femmes, mais à un véritable souci que le rituel se déroule correctement. Le mécanisme qui régit la vision du monde de Stepan semble concentré sur l'ordre rituel précis, sans lequel la cérémonie ne fonctionnerait pas : les dieux ne recevraient pas le message et les gens risqueraient de souffrir en conséquence des erreurs faites dans le cours du rituel.

Lors du rituel de 1996 à Num-to, les questions touchant aux rôles des sexes étaient imprégnées par la signification sacrale particulière de la cérémonie. Stepan attira mon aussi attention sur le rapport $\mathrm{du}$ lac et des îles sacrées qu'il contient avec les comportements rituels et les normes qui les régissent :

Dans ce lac, il y a un goulot d'étranglement, comme le cou de l'homme ou d'un animal. [En continuant] on arrive à la tête. Et dans cette tête, il y a deux lieux sacrés. Jamais femme n'y a mis le pied. Ce sont les yeux, ils sont comme des yeux. Ce sont deux îles avec des bouleaux, [il y pousse] six bouleaux, il y a deux yeux dans le lac sacré. Au milieu du lac, à quatre kilomètres du village, il y a une île qu'on considère comme son cœur. Quand on regarde le lac d'en haut, d'avion ou d'hélicoptère, c'est comme un animal vivant. Un cœur, une tête, des yeux, il y a aussi des touffes de poil. Les ruisseaux en sortent comme des touffes de poils.

Cette interprétation de Stepan, qui voit le lac Num-to comme un être sacré anthropozoomorphe, est intéressante. Il m'expliqua une fois de plus l'importance de l'île en forme de cœur, en soulignant que seuls les hommes avaient accès à cet endroit :

[Une île] se trouve au milieu du lac sacré. Elle est considérée comme sacrée parce qu'elle est comme le cœur du lac. Là, sur ce lieu sacré, jamais femme n'y est allée. Seuls les hommes le fréquentent. Cette fois-ci nous avons déplacé le lieu de la cérémonie au bord du lac, à un endroit où tous peuvent venir en famille, avec les enfants. Tous peuvent y venir avec leurs femmes.

19 Avant la cérémonie, à Num-to, les femmes se demandaient si elles allaient y être associées. Si la cérémonie avait eu lieu sur la grande île sacrée, elles n'auraient pas été prises. Il avait été question d'y tenir le rituel, mais cette solution avait été écartée.

La petite île n'était pas aussi sacrée et n'était pas un lieu réservé seulement aux hommes. Ceci explique la présence des femmes. Et l'objectif du rituel était suffisamment général pour favoriser leur participation. En même temps, cette cérémonie était aussi guidée par le besoin de trouver des relais vers l'extérieur. Sur un lieu sacré ouvert, il était possible d'accueillir tous les invités et les représentants des associations d'autochtones, parmi lesquels il y avait des femmes. Ainsi les participants à la cérémonie pouvaient-ils, aussi bien concrètement que symboliquement, représenter le peuple tout entier. En même temps, les femmes n'avaient pas toujours accès au lieu sacré au bord du lac. La cérémonie précédente avait été tenue, comme me l'a dit Sergej Griškin, un Khanty de la chaîne de télévision "Jugoria », sans femmes. À part cela, le 
rituel avait été semblable : trois rennes avaient été sacrifiés aux dieux, un attelage complet.

21 Stepan plaçait également la spécificité des lieux sacrés de Num-to dans un contexte géographique plus large, et tenant compte de différentes possibilités d'accès des différents groupes :

Tous les clans n'ont pas leur propre lieu. Les lieux comme le lac de Num-to sont très anciens. Chaque fleuve a son nom et le nom du fleuve est aussi sacré. Le lac Num-to est pour les cérémonies de tous. Mais chacun chez soi, sur son territoire, n'a pas des places pareilles. On n'en avait pas plus dans le passé. Et aujourd'hui non plus.

[Quand quelqu'un arrive d'une autre région et a besoin de sacrifier un renne,] il en achète un en échange d'argent ou de vodka. C'est une coutume, on a alors l'obligation de lui en vendre un. Il peut se rendre sur place en avion on en train et il ne peut pas prendre [le renne] avec lui. Mais sur place, au village ou dans les campements, ou encore auprès d'un čum, il doit acheter un renne aux gens d'ici. Peu importe à qui il s'adresse, tout le monde lui en vendra un.

La description de Stepan montre que pour les autochtones, Num-to est très largement reconnu comme une zone sacrée. On voir également que certaines règles rituelles générales sont largement partagées, et que l'on se soutient pour garantir la possibilité de réaliser une cérémonie. C'est un fait dont il faut tenir compte en arrière-plan de la portée générale du rituel. Pour la population de la région de Num-to, cela n'a rien d'extraordinaire que les gens y viennent faire leurs sacrifices. Ce qui était hors $d u$ commun, c'est la polysémie de cette cérémonie, et surtout l'engagement politique puissant et la tentative d'informer largement l'opinion publique qui la sous-tendent.

Pour caractériser le lieu de la cérémonie il faut aussi suivre la symbolique des couleurs et sa mise en œuvre par les autochtones. Le jeu des couleurs rituelles traverse tout le déroulement de l'action et commence par le choix de l'endroit et par sa préparation. Sur les bouleaux étaient accrochées des peaux de rennes avec la tête et les bois, qui remontaient aux cérémonies antérieures. On trouvait aussi sur les bouleaux des morceaux et des rubans de tissu. Le bouleau est un arbre blanc qui, d'après la vision du monde des autochtones, est en relation avec les dieux blancs et avec le monde d'enhaut, où, d'après les croyances khantyes et nenetses, ces dieux demeurent. Stepan m'a expliqué la question des peaux de rennes et des tissus laissés en offrande sur le lieu sacré après les cérémonies en les mettant en rapport avec la règle d'après laquelle les choses résultant d'une activité rituelle sur un lieu sacré restent sur place (à l'exception de la viande, que tous les participants doivent emporter):

Ces peaux qui sont suspendues au bouleau sacré ont été mises là quand nous sommes venus pour la première fois sur ce lieu sacré il y a deux ans. Quand on y fait un sacrifice, la peau et la tête, avec les tissus, sont attachées à l'arbre, elles y restent à jamais. Ces peaux et les têtes avec les bois, on ne les emporte pas.

L'héritage matériel des cérémonies antérieures est devenu partie intégrante, naturelle et obligée, du lieu sacré. Les objets blancs qui y ont été laissés en accroissent le caractère sacré par nature (représenté par les blancs bouleaux). L'impression générale que laisse l'explication de Stepan confirme que certains objets rituels doivent être accumulés. Comme on le verra ci-dessous, au cours de la cérémonie on répétera des actes et on accumulera des objets, en les ajoutant sur le lieu, pour assurer le fonctionnement de certains pouvoirs sacrés ou encore assurer la solidité du lien avec les dieux. 


\section{Le début du rituel} ordinaires. On alluma un feu sur le lieu sacré, et on commença à faire bouillir de l'eau dans des seaux. Le bois pour le feu avait été apporté sur place depuis les domiciles des participants. Stepan m'expliqua que la présence du feu était d'une importance fondamentale pour le rituel :

On a fait un feu. Parce que le feu, la flamme, est le plus fort de tous les cent dieux. Ceci parce qu'on peut tout brûler dans un feu. Voilà pourquoi on a commencé par faire le feu. Le feu a duré tant qu'on a été là, avec les gens et tout. [On faisait attention] à ce que le feu ne s'éteigne pas, il devait être toujours allumé.

À noter un point dans le discours de Stepan, qui n'est pas sans ambiguïté, quand il affirme que le feu

est le plus fort de tous les cent dieux.

On ne comprend pas bien ici si le feu est vu ici comme un dieu, le plus puissant ou le plus fort, comme une substance particulière ou encore comme le moyen le plus sûr d'entrer en contact avec les dieux, que ces derniers ne peuvent ignorer... et qui sont les cent dieux ? Est-ce que ce sont les dieux du ciel (ce qui serait en fait curieux) ou bien le feu est-il la plus forte des divinités d'en bas?

Kirill Kanterov, un informateur khanty d'Andrew Wiget et d'olga Balalaeva, a expliqué la particularité du feu par le fait qu'il est le seul dieu que tout le monde voit tous les jours :

Le feu est le premier esprit visible (Wiget, Balalaeva 2011, p. 132).

L'exclusivité du feu peut être d'autant plus manifeste que son action est explicite, perceptible et intelligible pour tous.

Le deuxième acte du rituel fut de nouer au cou du renne blanc deux morceaux de tissu, l'un plus long que l'autre. Les deux étaient de couleur claire, à dominante de blanc. Stepan m'expliqua le principe du choix des pièces de tissu et leur rôle rituel, en soulignant l'importance de l'apport de tous les participants :

Le tissu doit avoir toujours trois mètres de long. Peu importe la couleur. Cette offrande va aux esprits, aux dieux du ciel. Maintenant, tous ceux qui ont apporté une pièce de tissu la nouent autour du cou du renne. Tant qu'il est en vie, il aura ce tissu au cou. Qui n'a pas de tissu laissera des pièces de monnaie. Ceux qui viennent pour la première fois, ou qui sont étrangers, hommes ou femmes. Seulement des pièces argentées, seules les pièces blanches conviennent, celles en cuivre ne sont pas autorisées. Et on laisse aussi des billets de banque là où on a fait le sacrifice. Qui n'avait pas pris de tissu, prenait dans sa poche son mouchoir propre et le nouait au bouleau.

Stepan affirme ainsi d'une part que la couleur du tissu ne compte pas, et qu'il n'y a pas grande différence entre laisser en offrande des pièces ou des billets. Mais par ailleurs il reconnaît, et nous pouvions nous y attendre, que dans le cas des pièces, il est important qu'elles soient de couleur argentée et que, pour ce qui est du tissu (le mouchoir), il doit être propre. Il est logique, si on fait une offrande aux dieux du ciel, que les gens utilisent des couleurs à dominante blanche et du tissu propre (même si Stepan dans ce cas n'a pas mis l'accent sur la couleur du tissu). 


\section{Introduction politique}

32 Avant le véritable début du rituel eut lieu la partie exclusivement " grand public » de l'initiative. Il s'agissait de discours adressés aux leaders régionaux et fédéraux ainsi qu'aux téléspectateurs. Ils étaient censés les aider à prendre conscience de la situation des peuples autochtones, et aussi leur montrer l'intention des autochtones d'utiliser le rituel qui allait avoir lieu et les comportements religieux quotidiens pour défendre leurs droits publiquement.

Les premiers à s'exprimer furent Juri Vella, la Khantye Maria Kuzminična VagatovaVoldina (rédactrice de Hanty jasang, journal en khanty publié à Hanty-Mansijsk, poétesse khantye, originaire de la région de Num-to) et le voisin de Juri Vella, Oleg Ajvaseda (Nenetse de la forêt comme lui), à l'intention du groupe de tournage de la chaîne de télévision "Jugoria ». Il fut question de la nécessité de ne pas développer l'exploitation du pétrole dans la région autour de Num-to, de la culture traditionnelle des autochtones. Juri Vella informa les media qu'il dédiait une femelle blanche de son troupeau de rennes au président de la Russie (à l'époque de la cérémonie, c'était Boris El'cin, mais le même renne et/ou ses successeurs seraient hérités par ses successeurs à la présidence), alors qu'oleg Ajvaseda en dédiait une au gouverneur de l'okroug autonome de Hanty-Mansijsk (Aleksandr Filipenko au moment de la cérémonie). Derrière cet acte, l'idée était que puisque dorénavant le président et le gouverneur étaient propriétaires d'une partie du troupeau de Juri Vella et d'Oleg Ajvaseda, toute atteinte à l'environnement mettait en danger les biens du président comme du gouverneur. Ci-dessous, les textes des discours :

Juri Vella: Nous sommes arrivés au bord du lac sacré, le lac de Num-to, qui se trouve sur un gisement de pétrole. Au bord de ce lac, qui nourrit plusieurs fleuves, en présence de ma parentèle, je veux faire cadeau d'un renne. J'ai déjà donné l'argent. J'achète à un membre de ma famille un renne pour le président de la Russie. Si Boris Nikolaevič El'cin souhaite, avant les élections, l'emporter, l'abattre ou le donner à quelqu'un, je suis prêt à le faire à tout instant. Si Boris Nikolaevič El'cin avant les élections n'emporte pas son renne de mon troupeau, après les élections le renne sera propriété de celui qui aura gagné le marathon présidentiel. D'après les croyances de mon clan, ce renne pourra vivre éternellement. Comment, me direz-vous? Quand il vieillira, il tombera malade et je sentirai qu'il se sent mal, je l'abattrai et il donnera de la viande. Je mettrai à sa place un renne jeune et fort de mon troupeau.

Mais toujours d'après les croyances de mon clan, ce renne peut disparaître à jamais. Il peut être victime d'un accident ou tomber d'un coup de l'arme d'un contrebandier du chantier pétrolier. Ce n'est que dans ce cas que le renne du président pourra disparaitre. Et il y a encore une possibilité pour qu'il meure ou disparaisse : c'est le cas où son pâturage est détruit par quelqu'un ou quelque chose et que ce renne n'aie plus de quoi manger.

Je donne ma parole aux présidents de la Russie, l'actuel et le futur, que je mettrai tout en œuvre pour que leur renne vive le plus longtemps possible. Et je fais serment que je ferai tous les efforts, de toutes mes forces et de toutes mes capacités, pour protéger les pâturages contre toute action destinée à les endommager, les pâturages où se nourrira le renne du président, c'est à dire les pâturages de mon clan.

Voilà tout ce que je voulais vous dire sur la berge de ce lac sacré, Num-to, en présence de ma parentèle, à vous, habitants de notre okroug autonome de HantyMansijsk. 
Oleg Ajvaseda : Je suis un habitant du conseil de village de Var'ëgan et je suis arrivé ici, au bord du lac sacré, du lac Num-to, pour faire un sacrifice sur ce lieu sacré avec mes compagnons. De même, je veux offrir au gouverneur de mon okroug autonome, au camarade Filipenko, un renne, mon renne personnel. Et ceci afin qu'il fasse attention à notre peuple, qu'il nous accorde un minimum d'attention.

Juri Vella : Tu veux sans doute l'adresser au gouverneur de l'okroug pour qu'il t'aide à protéger ton pâturage ?

Oleg Ajvaseda: Oui. Je le lui offre pour qu'il m'aide à protéger mon pâturage, mes rennes, et ce faisant il aidera son propre renne. Et venant sur place, il peut l'emporter à n'importe quel moment de mon troupeau, s'il en a besoin. Et le garder où cela lui convient.

Juri Vella : Et comment est ce renne?

Oleg Ajvaseda : Il est de couleur blanche.

Juri Vella : Une femelle?

Oleg Ajvaseda: Oui, une femelle, qui lui donnera une descendance. Et que le camarade Filipenko veille lui-même sur sa descendance.

Juri Vella : D'ailleurs, j'ai oublié de dire que le renne que j'ai déclaré appartenir au président de la Russie, ce renne est également une femelle. Il est ainsi fort possible que dans quelques années, il n'y ait plus un seul renne appartenant au président, mais plusieurs.

D'ailleurs, encore un petit détail. Les gens du pétrole de Kogalym se sont engagés à compenser les dommages causés à ma famille en nous versant le salaire mensuel moyen d'un travailleur du pétrole. Environ trois millions.

Or avant-hier on m'a apporté l'argent : un million ${ }^{2}$. Je comptais sur cet argent pour acheter au président un attelage complet, c'est à dire trois rennes. Mais je n'ai eu d'argent que pour un seul renne. Voilà la raison pour laquelle je ne dédie au président de la Russie qu'un seul renne.

Maria Vagatova-Voldina: Je veux vous dire que je suis venue ici avec une grande joie. Car c'est ici que s'est passée mon enfance ; ici, il y avait des rennes. Beaucoup, beaucoup de rennes. Mais maintenant, les gens sont agités et ils ont adopté une déclaration. Je pense que c'est une exigence, une demande juste pour que le lac sacré, le lac Num-to, cette terre soient laissés tranquilles. Parce que déjà s'élèvent des tours de forage. Nous, nous pensions que nous étions dans notre okroug, nous pensions que la terre de Num-to était toujours intacte. Nous découvrons qu'ici la douleur est aussi immense que dans les raïons de Surgut, de Nižnevartovsk, de Neftejugansk.

Merci, de ce que les gens ici, à cet endroit, aiment tout autant les rennes, tout autant que tous les hommes. Et ils ont donné au gouverneur et au président de la Russie les meilleurs cadeaux. Bien sûr, puisque nous n'allons pas à l'église, nous n'avons rien à y demander. Ici, c'est le seul lieu sacré qui nous est resté dans toute la toundra. C'est pourquoi je m'associe à tous les signataires de la lettre aux gouverneurs des deux arrondissements. Et [merci] à ceux qui aujourd'hui s'adressent au ciel et qui ont apporté les cadeaux les plus précieux, tout un attelage. Que Dieu veuille que nous tous, que nous tous souhaitions ne jamais en être privés. Juri Vella: Nous voulons confirmer notre acte devant nos dieux comme avec un tampon. Le sacrifice que nous ferons aujourd'hui, la consécration des rennes aux dieux, c'est l'acte d'offrande des ces rennes que nous confions au président de la Russie et au gouverneur. Et nous avons confiance en notre appel, celui que nous envoyons au gouverneur de l'okroug et qui lui dit que cette terre a besoin d'être préservée.

Dans le déroulement du rituel, ces discours se distinguaient clairement des autres activités. Ils étaient adressés aux caméras de la télévision, Juri Vella regardait vers elles et parlait au microphone, sans s'adresser au peuple. Les participants à la cérémonie n'ont même pas entendu, sans doute, ce qui s'y est dit. En même temps, les présents savaient déjà que des rennes venaient d'être offerts au président et au gouverneur. 
Mais probablement personne ne pouvait deviner de quelle manière exactement cet acte se présentait et comment Juri Vella dans son adresse faisait le lien entre la politique et la vision du monde autochtone. (La présentation des discours montre clairement qu'Oleg Ajvaseda agissait selon les instructions précises de Juri Vella, qui l'aida à formuler des positions essentielles.) Il s'agissait là d'un acte politico-sacral innovateur, jouant avec des éléments en même temps de discours politique et de rituel traditionnel, de vision du monde.

L'effet de ces discours et des actes de consécration n'a cessé d'être présent dans le discours autochtone sur les droits des communautés et n'a pas perdu de son actualité (cf. par exemple Prozes 2013). Or au cours du rituel que nous examinons, il était question d'un interlude, au cours duquel les autres activités étaient suspendues. Par la suite, on se concentra de nouveau sur le déroulement de la cérémonie. Juri Vella et Oleg Ajvaseda redevinrent des participants ordinaires à la cérémonie, Maria VagatovaVoldina retourna avec les autres femmes à l'arrière-plan et les journalistes de la télévision filmèrent le reste des activités en qualité de spectateurs, sans contraindre les participants à adapter leurs activités aux besoins de la caméra.

\section{La collecte des offrandes}

Après la consécration des rennes aux hommes politiques et les discours tournés vers le grand public le rituel se poursuivit sous sa forme traditionnelle. La présence plus importante qu'à l'accoutumée de femmes et l'activité des journalistes de la télévision ne manquèrent pas de laisser quelques traces sur la manière dont le rituel se déroula : il fallut adapter le positionnement et les mouvements des femmes à ce rituel centré sur les hommes; opérer devant la caméra intimida quelque peu les hommes qui menaient le rituel, rendant leurs mouvements plus raides et leur attitude plus sérieuse. Mais la multiplicité des éléments et la clarté des symboles du rituel cadraient suffisamment leur activité, de sorte que la plupart des participants étaient occupés à assurer le déroulement de la cérémonie. C'est ainsi que les procédures ne furent de manière générale guère gênées de cette part de formalité supplémentaire.

Quand les discours furent terminés, on étendit un tissu sur la neige entre les cèdres et les sacrificateurs, et on y posa les offrandes. Chacun mit ce qu'il ou elle avait apporté : des biscuits secs, des snickers, des chocolats, des papirosses "Prima », de la vodka, du thé. Stepan fit une remarque sur la manière de déposer les offrandes : il estimait que la présence du tissu était une bonne chose, mais il aurait aussi accepté si, faute de tissu, les offrandes avaient été disposées directement sur la neige. C'est là l'une des rares occurrences où Stepan ne fit pas preuve de sa rigueur ordinaire pour reconnaître la légitimité de différentes options :

Ils ont mis les bouteilles sur la neige. Je regardais. Mais moi j'avais pris un tissu à la maison. C'est un reste, il était suspendu à un clou, je l'ai pris. Ensuite je l'ai mis sur la neige. Alors, ils ont mis les bouteilles dessus. Ils les avaient mises sur la neige, sur la neige propre. Ils n'avaient rien pris. C'est autorisé, bien sûr. On met la bouteille et le petit verre pour la vodka directement sur la neige. Mais moi, je connais nos coutumes. C'est pourquoi j'ai pris le tissu rouge avec moi en venant dans la forêt. Il était long de moins d'un mètre.

Les bouteilles de vodka étaient au nombre de sept, c'est à dire, en fait, trois bouteilles d'un litre et une "demie » (en général, le prototype est la bouteille d'un demi-litre, 
c'est pourquoi les bouteilles d'un litre comptaient pour deux). C'est ce que Stepan confirmera plus tard au cours de l'entretien :

Nous avons sacrifié à sept esprits, sept divinités. Nous avions sept bouteilles. Exactement sept. Les grandes, n'est-ce pas, c'est [comme] six [petites bouteilles]. Et la septième était une petite. Voilà comment nous sommes arrivés exactement à sept.

Il était essentiel qu'il y ait sept bouteilles. On comprend facilement l'analogie avec les conceptions cosmologiques traditionnelles. Pour les Ougriens de l'Ob' et pour les Nenetses, le sept est le chiffre le plus important: on croit que le nombre de dieux blancs vivant au ciel est de sept, comme le nombre de strates dans le monde d'en-haut (Rombandeeva 1993). Les gens venus à la cérémonie n'ont pas particulièrement essayé d'avoir un nombre de bouteilles équivalent au nombre de dieux ou de strates sacrées. Ils ne s'étaient pas mis d'accord sur le nombre de bouteilles. Sur place, ils se rendirent compte que la situation pouvait être interprétée comme reflétant le fait que les participants avaient apporté précisément autant de vodka qu'il fallait. D'une part cela illustre le fait que la coïncidence est une bonne chose et que ce qui se passe est discrètement approuvé par les divinités (cf. Novikova 1999a). Par ailleurs cela reflète la stratégie rituelle flexible des autochtones - si la coïncidence avec l'harmonie cosmologique ne saute pas aux yeux, on peut trouver une interprétation appropriée et découvrir l'approbation bienveillante des dieux dans un message plus obscur et ambivalent.

\section{Les positionnements pendant la cérémonie}

En plus du caractère sacré en général de la région et de ses liens avec la topographie globale de l'univers mental autochtone, d'autres éléments ont une importance considérable dans le déroulement du rituel: le positionnement des hommes et des rennes. Cette microtopographie sacrale a pour fonction, dans la perspective du déroulement du rituel, de garantir l'harmonie de l'ensemble.

Les rennes à sacrifier furent détachés de l'arbre et disposés en rang, la tête tournée vers le sud, vers le soleil, vers le monde d'En-haut, vers les dieux blancs, mais aussi vers les offrandes et les cèdres. Les hommes étaient debout en arc derrière eux, et les femmes, derrière les hommes, rassemblées vers l'ouest: elles restaient à l'extérieur de l'axe rituel mental sud-nord (cèdres-offrandes-rennes-hommes-lac Num-to). Pour Stepan, cette question du positionnement était suffisamment importante et compliquée pour donner lieu à une explication détaillée :

Or [on prie] les dieux blancs, les dieux du ciel, toujours en direction du soleil. Quand on prie le dieu noir, Hyn-iki, on le fait en direction de l'aube. Alors le renne doit être placé dans cette direction. Les jeunes ne le savaient pas. Ils les avaient mis devant nous [la tête en direction du nord]. Mais le plus âgé des vieillards de NumTo, à la tête toute blanche, Täkvjak, soudain il s'en est souvenu. C'est lui qui a dit de mettre les rennes la tête vers le soleil. Au début, les jeunes avaient mis les rennes dans l'autre sens. Dans notre direction, vers la nuit. Pour qu'ils soient vraiment morts. Or nous avons prié avant tout pour la vie, c'est pour la vie que nous avons prié. Si le vieillard ne l'avait pas dit aux jeunes, je l'aurais dit moi-même, qu'il faut mettre la tête en direction du soleil. Or les jeunes les ont mises en direction de la nuit. Si on veut s'adresser à la nuit, au dieu noir, on peut faire le sacrifice au village. Nous n'aurions pas dû absolument venir ici [sur le lieu sacré]. Nous aurions pu rester au village. C'est qu'on pense que les dieux, les dieux noirs et ceux du ciel, ils 
sont fâchés. Le dieu noir [agit], quand ils boivent beaucoup de vodka, ils se saoulent.

Il arrive que l'un en tue un autre, que l'un d'entre eux aille en prison. sacrifices aux dieux noirs - et dans ce cas les animaux "meurent réellement ", ce qui voudraient dire que les animaux sacrifiés aux dieux du ciel ne meurent pas et renaissent ? Stepan a senti la nécessité de revenir sur cette question et d'expliquer plus en détail les circonstances des sacrifices faits aux dieux noirs :

Les cèdres sont consacrés au dieu noir, Hyn-iki, auquel on fait aussi des sacrifices. Jadis les vieillards, nos ancêtres, venaient aussi sur la rive là-bas [faire des sacrifices] auprès des sept cèdres. À cinquante mètres de l'endroit où nous avons fait la cérémonie, au bord du lac, il reste encore un vieux cèdre. Et il en poussé six, des jeunes. Et voilà les sept cèdres. Là aussi on fait des sacrifices, au dieu noir. Sur ce même lac sacré. Le dieu noir, il est rattaché à un arbre noir, le cèdre. On y suspend aussi des morceaux de tissu. On ne lui sacrifie qu'un renne noir. Alors qu'on offre au dieu blanc un renne entièrement blanc. La raison pour laquelle [on fait des sacrifices] au dieu noir est pour que les gens ne soient pas malades, ne meurent pas. On prie de la même manière qu'au lac du ciel. On fait aussi des sacrifices. Ce sont aussi des rennes, de la vodka, des aliments. On dresse aussi une table.

Comme on le voit, il n'y a pas grande différence dans les éléments du rituel de sacrifice, qu'il soit adressé aux dieux blancs ou aux dieux noir (si l'on ne tient pas compte de la symbolique des couleurs, du choix de l'endroit et de l'orientation spatiale - différence qui fonctionne suivant le principe de la symétrie, avec un renversement des éléments fondamentaux du rituel, à l'image de la structure du monde). Stepan met l'accent sur le 
fait que la cérémonie se déroule de manière identique, de même que le positionnement des offrandes de nourriture et de boisson.

En même temps, on ne comprend pas particulièrement pourquoi on sacrifie les rennes aux dieux blancs le regard tourné vers les dieux noirs. Peut-être ce fait renvoie-t-il à une certaine symétrie ou harmonie entre les univers des dieux blancs et des dieux noirs. C'est peut-être aussi un simple hasard (que sept cèdres aient poussé juste à cet endroit-là), mais les autochtones traitent ce type de hasard en lui donnant du sens voire de la prédétermination (comme par exemple l'interprétation du nombre des bouteilles de vodka; sur l'interprétation de la fatalité dans la vision du monde des habitants autochtones de cette région, cf. Kulemzin 1980, p. 107-112).

L'expression « on prie à ce lac du ciel » fait référence au fait que le lac incarne le dieu du ciel, ou encore l'univers de tous les dieux célestes; dans ce cas, les cèdres peuvent représenter le renversement de ce modèle, le lien avec le monde d'En-bas (cf. ci-dessus l'explication de Stepan sur l'anthropo-zoomorphie du lac Num-to, sur ses îles et sur sa nature).

49 Avant le début de la prière, on versa de la vodka. Les rennes étaient disposés sur un rang, le plus à l'est étant un renne blanc, un mâle. Stepan m'expliqua qu'il s'agissait du chef de l'attelage, celui qu'il fallait tout d'abord tourner vers les dieux blancs. Les autres rennes de l'équipage suivraient.

\section{La prière du sacrifice}

Certains hommes (Täkvjak, Juri Vella, Oleg Ajvaseda, Stepan Randymov), au moment de prier, se découvrirent. La plupart des autres gardèrent leur couvre-chef. Tout d'abord, Täkvjak récita une longue prière. Puis Oleg Ajvaseda récita lui aussi une prière, plus courte. Plus tard, Stepan m'expliqua le contenu de la prière et son contexte, ce qui avait déterminé la nature de celle-ci :

[Aujourd'hui on a adressé le sacrifice] à ce lac sacré. Num-to, le lac du Ciel, c'est pourquoi c'est un lac sacré. [Nous avons demandé] que la vie des gens d'ici, des autochtones, de tous [soit bonne]. Que tous les enfants vivent tranquillement et que les adultes puissent vivre dans tous les endroits ou leurs pères et mères ont marché. Qu'ils n'oublient pas [ces endroits].

Mais ceux qui veulent que leurs enfants les oublient, c'est leur affaire. Eux, ils n'écoutent pas, ils ne croient pas non plus en dieu. Et on n'oblige pas les enfants par la force. Ceux qui veulent, écoutent leurs parents. Il y a des jeunes qui sont venus ici. Cela fait cent ans que leurs parents ne vivent pas. Ce sont eux les héritiers. Pour qu'eux aussi viennent à cet endroit faire des sacrifices. Au même endroit. Là où allaient leurs grands-pères. Où nous avons été aujourd'hui. Ce sacrifice était pour la vie de famille, pour que les enfants vivent et soient en bonne santé. Pour qu'ils aient de la chance avec les rennes. C'est que tous les Nenetses vivent avec les rennes.

Le vieux Täkvjak, le vieillard grisonnant parmi les Nenetses des forêts, est le plus âgé qui est resté de sa génération. C'est pour cette raison qu'on lui a confié [la prière], [pour qu'il prie] à tous les dieux, les esprits du ciel du lac sacré, pour qu'ils prennent bien tous les rennes dans leur main. En plus des rennes, il y avait aussi d'autres offrandes. Tous ceux qui sont venus - certains ont apporté de la vodka, d'autres des aliments. Ils ont tout préparé sur la table. Sept bouteilles de vodka et sept petits verres de vodka. Pour que ces dieux célestes du lac sacré, pour que tous les esprits acceptent avec bienveillance tout ceci. Il a parlé de tous les dieux, de tous les dieux du ciel qui existent, tous. Cent dieux, autant qu'ils sont, tous, il a invité 
chaque dieu individuellement. Kaltaš-imi ${ }^{3}$... Il y en a beaucoup, on ne peut pas tous

les compter.

51 Les prières étaient de nature générale : on y demandait une bonne vie pour tous, beaucoup de rennes, de la paix pour les enfants et la possibilité de vivre là où vivent leurs ancêtres ; les prières demandaient aussi que les enfants n'oublient pas les dieux. Le caractère général des prières était dû à une certaine imprécision des objectifs sacraux du rituel. Il y avait beaucoup de présents et il fallait demander quelque chose qui concerne tout le monde. C'était aussi ce qu'il y avait de plus facile et de plus sûr demander une bonne vie en général. Par ailleurs, le rituel était politique, il y avait une dimension tournée contre l'industrie du pétrole et les évolutions politiques y afférant. La tentative de relier une initiative destinée à défendre les droits des autochtones avec un rituel de sacrifice à tous les dieux blancs risquait de susciter de la confusion chez les participants, du fait du caractère innovateur de sa dimension politico-religieuse. Donc, il était naturel qu'on demande aux dieux beaucoup de rennes, ce qui entre autres pouvait contenir une allusion au fait que les flots de pétrole ne permettent pas la chance $^{4}$ dans ce domaine.

Quelques jours plus tard, Stepan soulignera que les prières avaient été entièrement couronnées de succès, comme le montrait le fait que le lendemain du rituel il avait fait beau :

Quand nous sommes partis à la cérémonie, il faisait mauvais; maintenant il fait clair. Nous avons demandé que le temps soit beau, quand tu prendras l'avion. Nous avons fait ce sacrifice pour que le temps soit beau quand les gens repartiront [en avion]. Et encore que les gens qui sont venus ici aient dans leur vie de la chance sur le chemin. Il arrive qu'il y ait du vent, des tempêtes. C'est une habitude que nous avons. Et si une famille ou une personne a été touchée par le malheur, nous [demandons] de la chance pour eux aussi.

Quand nous [demandons] pour tous, alors c'est que pour la personne qui est venue ici il y ait du beau temps pour repartir, en avion ou en hélicoptère. C'est pourquoi c'est bon que tous les esprits et les divinités auxquels nous nous sommes adressés se réjouissent.

Le temps, quand on voyage, est d'une importance existentielle, mais dans l'interprétation de Stepan il est aussi important à plus long terme ou métaphoriquement ("qu'ils aient dans leur vie de la chance sur le chemin »). D'après lui, les dieux ont fait comprendre, en envoyant du beau temps, qu'ils étaient bienveillants vis-à-vis du rituel et des prières de la veille.

\section{Le sacrifice des rennes}

Après la prière, on abattit les rennes. Deux Nenetses tenaient un lasso autour du cou du renne ; ils tirèrent dans des directions opposées. Un troisième Nenetse frappa le renne sur la nuque avec le dos de sa hache. Avant, il simula le coup deux fois et ne le porta vraiment qu'à la troisième. Le premier renne tomba au début du côté gauche. Rapidement, les Nenetses le tournèrent sur le flanc droit. Cette manière de faire les choses dérangea dans une certaine mesure Stepan, qui fit remarquer que l'animal sacrificiel doit toujours tomber du côté droit. En même temps, quand cela arrive, il n'y a pas d'autre solution, il faut le retourner.

En tombant du côté droit, le renne transmet l'acceptation par les dieux de ce sacrifice. Mais il est aussi possible de peser sur le résultat en tournant l'animal mal tombé du bon 
côté. Comme cet acte semble être accepté, la manière de tomber semble ne pas être une information univoque sur l'état d'esprit des dieux; si les hommes retournent l'animal, c'est un signe de bonnes intentions de leur part. Il se peut aussi que l'on assiste à quelque chose comme une négociation. Les dieux au début rejettent le sacrifice, mais les hommes le reproposent et alors ils sont satisfaits (car le renne finalement reste couché sur le flanc droit).

Les deux rennes restants, après avoir reçu le coup sur la nuque furent poussés de manière à tomber immédiatement du côté droit. Une fois que les trois rennes furent à terre, on leur enfonça un couteau dans la nuque et dans le cœur. Puis, on tira le troisième renne mort de manière à ce qu'il fasse un tour dans le sens des aiguilles d'une montre. Les trois rennes furent alignés placés côte à côte, la tête tournée vers le sud. Stepan était satisfait de la manière dont les Nenetses avaient opéré, elle correspondait à sa conception d'un rituel bien mené :

[On fait tourner les rennes sur la neige] une fois dans le sens des aiguilles d'une montre. Le vieux grand-père blanc, grisonnant, Täkvjak, dit aux jeunes de faire faire [aux rennes morts] encore un tour dans le sens des aiguilles d'une montre. Comme le soleil tourne. C'est la coutume de faire un tour dans cette direction lors de ces sacrifices sacrés. Il a prié tous les dieux, tous les esprits et ensuite il doit faire trois tours dans le sens des aiguilles d'une montre.

57 Les rennes morts furent disposés sur la neige comme dans un attelage. À gauche, le renne blanc, le renne de tête. Les pattes étaient tournées vers l'est. Sa tête fut tournée à $90^{\circ}$ (le museau tourné vers le ciel) et les bois enfoncés dans la neige. Puis, Stepan Randymov les frappa de son lasso sur le flanc et émit des sons comme pour les encourager à bouger. Là-dessus le renne blanc tressaillit, ce qui voulait dire que les dieux blancs l'avaient accepté. Le dernier renne lui aussi trembla un peu sous le coup du lasso, mais Randymov n'en tint pas compte. En fait, il n'avait pas vu le mouvement du troisième renne, parce qu'il lui avait tourné le dos avant. Il expliqua son acte, qui était nécessaire pour que les rennes meurent effectivement et soient envoyés auprès des dieux blancs :

[Pour les envoyer] chez les dieux d'En-haut, il faut que la tête des rennes soit tournée vers le soleil. Il ne faut pas [la tourner] vers la nuit. Nous les avons disposés en attelage. Celui avec les bois était le chef. Lui, il a été bien accepté. Même après que je l'ai frappé trois fois avec le lasso, il a bougé trois fois, alors qu'il était mort. Mais les deux sur les côtés, ils sont tombés et ils étaient aussitôt morts. Le chef de l'attelage a été aussitôt accepté, dès que nous avons prié les esprits. Tous ceux qui ont été touchés [avec le lasso], ces rennes doivent bouger trois fois avec leur cœur, pour ne pas tomber morts tout de suite. Il faut absolument les frapper trois fois avec le lasso, pour qu'ils bougent. Après le renne reste immobile et mort, mais [seulement] après que vous l'avez frappé trois fois avec le lasso.

Les frémissements montrent que le sacrifice est accepté par les dieux. Cela voulait dire que la chute du renne du mauvais côté n'était pas un mauvais signe définitif, et que les dieux ont fini par accepter l'offrande, parce que tous les autres actes ont été réalisés de manière correcte.

«Tomber aussitôt mort » et "être vraiment mort » sont des expressions que Stepan utilise quand quelque chose au cours du rituel va de travers. Cela renvoie sans doute à l'idée qu'ont les autochtones que l'animal sacrifié, au cours d'un rituel qui se déroule correctement, meurt peu à peu. Pour ce faire, il faut que toutes les étapes soient bien menées, pour qu'il n'arrive rien au renne sur son chemin en direction des dieux blancs, alors qu'il est dans cet état "pas réellement mort», où il convient de le maintenir 
quelque temps. Il n'est pas exclu qu'en cas d'erreurs fondamentales dans le rituel, la victime puisse se retrouver non pas chez les dieux blancs, mais chez les dieux noirs. C'est la conclusion qu'on peut tirer du discours de Stepan.

Après l'abattage des rennes et leur disposition alignée sur la neige, les hommes se placèrent de nouveau derrière les rennes, le visage tourné vers le sud, les femmes et les invités à l'ouest par rapport aux rennes, le visage tourné vers l'est. Ils se mirent alors à crier fort sept fois, et ils tournèrent sur eux, tout en s'inclinant aux quatre points cardinaux. Tout ceci était assez confus, de sorte que tous ne firent pas le même nombre de tours et de révérences. D'après Stepan, il aurait fallu faire chacun de ces mouvements sept fois :

On a appelé tous les dieux, tous les esprits à venir sur place. Peut-être certains n'ont pas entendu l'appel. Celui-là n'a pas été appelé. Après on crie sept fois. Et [il faut faire] sept tours après les sept cris. De tous les dieux qu'il y a sur la terre, les plus importants sont les dieux blancs du ciel. Comme le père et la mère et le soleil, sept fils, sept filles. Pour cette raison sept fois. Lors des autres sacrifices on ne crie pas sept fois comme ici. Seulement trois fois. Quand on n'appelle pas tous les dieux. Mais maintenant, tous les dieux qui sont sur la terre et dans les cieux, on les a tous appelés. [Et on leur a sacrifié] tout un attelage de rennes.

61 Comme le montre l'interprétation de Stepan, les sept cris et les tours représentaient la consécration du sacrifice à toutes les principales divinités. Si on tient compte du fait que d'après lui tous les dieux sur terre étaient attendus, il n'est pas étonnant qu'on puisse envisager qu'une partie des divinités, vu le peu de cris et de révérences, puisse avoir ignoré l'invitation.

Dans la vision du monde des autochtones, les dieux sont caractérisés par une certaine paresse, par leur ignorance et par leur tendance à s'amuser aux dépens des gens. Par exemple, les Khantys du Kazym connaissent le dieu Rat ole foyer'), qui sert « de messager, quand on s'adresse à un dieu avec un sacrifice. Si le dieu n'est pas là, qu'il a été appelé ailleurs ou qu'il vaque à ses occupations, Rat ole voir un autre dieu et si lui aussi est occupé, il va en voir un troisième, jusqu'à en trouver un qui a le temps de répondre à la demande » (Semjon Juhlymov, 55 ans, TT1992).

Le sens de l'humour des dieux ressort bien de l'histoire qu'a racontée Juri Vella, quand en août 2013 il a expliqué ce qui se passe quand les Nenetses demandent à Numi-Torum de la pluie :

Torum veut montrer qu'il peut tout faire. Il fait tomber tous les jours dix gouttes de pluie et dit aux gens : "Vous voyez? Je vous ai donné dix gouttes de pluie! Ne dites pas que je ne vous ai pas donné de pluie! Je vous en donne tous les jours! Je suis tout-puissant!"

Dans ce contexte, on comprend que les autochtones aient tenté de faire comprendre aux dieux leurs désirs et intentions sans ambiguïté, aussi clairement et aussi énergiquement que possible, et de faire en sorte qu'ils ne puissent échapper à leur demande. Il n'était pas question de hurlements grossiers, mais de l'application d'une stratégie de communication choisie en tenant compte du caractère des dieux.

\section{Le lasso dans les branches}

Après avoir prié, les responsables de la cérémonie se couvrirent la tête de capuchons ou de bonnets. Les lassos qui étaient autour du cou des rennes sacrifiés furent jetés dans 
les branches des bouleaux. Deux y restèrent suspendus, le troisième tomba par terre. Pour Stepan, c'était là encore un des éléments incontournables du rituel :

Ces lassos, avec lesquels les rennes étaient attachés, on les jette [dans les branches des bouleaux]. Pour que grâce à ces trois rennes, tous les esprits, tous les dieux [tournent leur attention] là, [où] les gens ont encore des rennes. [Pour qu'ils aient] de la chance avec le troupeau, que les rennes grandissent, qu'ils vivent bien. [Pour qu'ils] aient de la chance de manière générale, à la pêche, à la chasse. Pour que si quelqu'un va quelque part chasser, attraper des animaux, pour que chaque bestiole vienne à sa rencontre. Là, il n'y avait pas d'arbres, alors ils les ont jetés là-bas [dans les fourrés]. Le lasso doit absolument être jeté sur un arbre. Peu importe lequel. Il suffit qu'il soit près.

D'après l'explication de Stepan, les rennes abattus à ce moment-là sont déjà avec les dieux et, il faut l'espérer, c'est la puissance des dieux qui passe, par l'intermédiaire du lasso, dans le monde des hommes et des animaux, favorisant la multiplication des rennes, leur santé, le succès à la chasse et à la pêche. Il faut aussi mentionner que ce sont les esprits qui envoient aux chasseurs le gibier. L'efficacité de l'enchantement est garantie par le fait que le lasso reste suspendu aux arbres. Que l'un soit tombé est mauvais signe.

\section{Le repas sacrificiel}

Puis on se mit à manger les offrandes apportées. Ce sont les Nenetses qui commencèrent, et Täkvjak distribua la vodka avec les petits verres. Chaque présent eut droit à un verre. Le petit verre passait d'une main à l'autre dans le sens des aiguilles d'une montre. Täkvjak gardait la bouteille. C'est lui qui versait la vodka dans le verre et il le remettait à un participant qui le vidait et le lui rendait. Il le remplissait à nouveau de vodka et remettait le verre à la personne suivante, celle qui était assise, à genoux ou accroupie à gauche de la première. Les hommes commencèrent, puis ce fut les femmes qui allèrent manger et prendre de la vodka. Stepan mit en rapport l'acte rituel du repas avec le fait que tous les esprits et les dieux étaient invités, donc tous devaient être là :

Là on vient d'inviter tous les esprits, tous les dieux. Et on [leur a mis] la table avec les offrandes des rennes. Tout d'abord, les verres. Sept verres, sept bouteilles. Il faut que le nombre ne soit pas pair, quatre, ou six, ou deux. Dans les verres il y avait de la vodka. Le premier il faut le donner au feu, au dieu du feu. Tous doivent boire. On commence tout d'abord avec les hommes, on leur donne un verre. Quand tous les hommes ont pris les verres qui étaient sur la table au moment où on a appelé les dieux, vient le tour des femmes.

Au début du repas, Stepan avait apporté un verre de vodka qui avait été rempli avant et se trouvait sur la table au moment du sacrifice et il l'avait versé dans le feu, puisque celui-ci, disait-il, «était le plus puissant des cent dieux ». En même temps, il avait dit une prière en russe. Les souhaits exprimés étaient très généraux - que tout aille bien, que tous soient en bonne santé et que tous retournent bien chez eux (beaucoup de participants étaient venus de loin, pas seulement de la toundra autour de Num-to). Stepan pria en russe pour que tous comprennent (il était filmé en même temps par la télévision). De plus, comme il m'expliqua par la suite, les dieux comprennent toutes les langues. Après la prière, Randymov s'inclina en direction des quatre points cardinaux, faisant en même temps trois tours sur lui. Il versa la vodka dans le feu nu-tête.

Le contenu de la prière de Stepan était le suivant : 
Au dieu le plus fort, au feu... que vos enfants, tous... que tout le monde soit en bonne santé, que les invités qui sont venus ici retournent avec un temps propice, qu'ils soient en bonne santé. mentionnées ci-dessus - pour garantir la réalisation des vœux exprimés, pour garder le grand nombre de participants en situation de sécurité sacrale et pour éviter les mauvais tours que les dieux peuvent vous jouer en cas de demande présentée de manière trop discrète (réponse à la demande au pied de la lettre, qui risque de ne pas satisfaire les gens, comme évoqué ci-dessus).

\section{Le dépeçage des rennes}

Quand les hommes eurent mangé, ils se mirent à écorcher les rennes et à les dépecer. Ils mirent de côté les peaux avec les têtes (sans la mâchoire inférieure) - mais toujours avec le nez plus ou moins tourné vers le sud.

Avant de commencer à écorcher les rennes, ils prirent le tissu qui avait été noué au cou du renne de tête et l'accrochèrent aux branches du bouleau. Plus tard, avant de mettre les peaux et les têtes sur la neige, les pièces de tissu furent renouées à la tête coupée du même renne.

Stepan Randymov prit, avec un bol, du sang dans le ventre ouvert des rennes et le jeta en diverses directions, loin du lieu du sacrifice, surtout en direction du sud, vers les cèdres, sur un arc allant du sud-est au sud-ouest. Stepan jeta le sang sept fois. Il expliqua lui-même son acte une fois de plus par la volonté de donner satisfaction aux plus importants des dieux et par le besoin de suivre la coutume de manière rigoureuse, comme dans le cas des actes précédents dédiés de même aux sept dieux :

J'ai pris [du sang] des trois rennes, [je l'ai jeté] à sept endroits. Plus de sept, on n'a pas le droit. Quand nous avons pris les verres de vodka, c'était pareil [ils étaient sept]. Pour les esprits, pour les dieux, tout ceci est pour eux. Nous avons prié les esprits. On n'a pas le droit [de jeter le sang] plus de sept fois. [On a sacrifié] les rennes à sept dieux ou esprits, parce que nous avions sept bouteilles. [J'ai pris] du sang de trois rennes. J'ai vu que personne ne voulait [le faire]. Sans doute les jeunes ne savent même pas. Je [garde] mes coutumes, comme mes parents me les ont enseignées. Et j'ai tout fait moi-même.

Les morceaux de viande restèrent sur la neige, une partie d'entre eux furent emportés dans des seaux auprès du feu et on les fit bouillir. En même temps le repas se poursuivait. C'est la partie du rituel la plus longue, au moins deux heures.

\section{Entre-temps}

Bien que la plupart des hommes fussent occupés à dépecer et faire cuire la viande de renne, cette phase du rituel était la moins intense. L'ambiance qui commença à dominer était celle de la détente. Des Khantys arrivèrent, Aleksej Moldanov avec ses fils Timofej et Grigorij. Ils venaient en traîneau d'un endroit à 54 km du Kazym. Ils avaient aussi apporté une bouteille. Aleksej discutait avec tout le monde activement, il intervint à la télévision et chanta longuement.

Le moment le plus tendu (même s'il échappa sans doute à la plupart des présents) fut quand Täkvjak commença à chanter. Il demanda à la télévision de le filmer tout 
particulièrement puisque ce chant était pour lui très important. À côté de Täkvjak se tenait Stepan Randymov nu-tête; il regarda tout le temps le Nenetse attentivement, tout en s'inclinant de temps en temps vers le sud. Stepan m'expliqua l'importance de la situation aussi bien pour les Nenetses de la région et à un niveau plus personnel :

Le vieil homme se mit à chanter pour que les jeunes après sa mort chantent ses chants de manière identique. Puis il a parlé des rennes. De comment nous les avons sacrifiés. Comment nous avons mis la table. Avec ce chant, de nouveau [il s'est adressé] à tous les dieux, il a invité tous à nous rejoindre.

Tant qu'il chantait, je le regardais tout le temps dans les yeux. Je suis Khanty. Ma femme est nenetse. [J'avais peur] qu'il ait l'idée de faire quelque sorcellerie contre moi. [C'est pourquoi] je voulais écouter. D'un œil, le gauche, il ne voit pas. Mais des larmes coulaient de l'œil droit. Il a chanté. C'est pourquoi j'ai regardé son visage et il chantait, mais dans son âme il sentait qu'il n'avait plus longtemps à vivre au bord de ce lac.

77 Ainsi Stepan pensait que Täkvjak avait voulu, à l'aide de la télévision, préserver son chant pour les générations de Nenetses à venir. En même temps, ce chant était un message aux dieux, pour assurer une fois de plus le succès de la cérémonie. Curieusement, le chant de Täkvjak avait un potentiel de sorcellerie, que Stepan tenta de capter et même de réduire à l'impuissance. Cette possibilité d'ensorcellement provenait peut-être de l'âge de Täkvjak, qui le mettait en position liminaire entre divers mondes. En même temps, tous les participants au rituel se situaient dans un certain sens en contact avec le monde des dieux et des esprits. Qu'au cours du rituel tourné vers les dieux blancs il soit facile de se retrouver dans la sphère d'influence des dieux malveillants, des esprits mauvais et des dangers qu'ils représentent, cela apparaît clairement dans les commentaires de Stepan sur le positionnement des rennes et le problème des dieux noirs.

\section{Laisser sur place les bouteilles, manger la viande sacrificielle et former les jeunes Nenetses}

Les bouteilles vidées ont été mises par les Nenetses, sur initiative de Stepan Randymov, au bout des branches des arbres. Stepan explique ceci par la nécessité d'éviter de laisser traîner des ordures. En même temps, il présenta aussi une explication rituelle à cet acte :

Dès lors qu'il y a un lac sacré. C'est pour les dieux, pour les esprits du ciel que l'on a vidé ces bouteilles dans la forêt. On a bu toute la vodka. Cette vodka était aussi [une offrande] pour ces dieux. Ils sont vivants, ils trouvent de la vodka partout. Si on laisse la bouteille vide, ils pourront la remplir. Je fais toujours comme ça.

Là, nous étions sur place, nous étions assis. [Les jeunes Nenetses demandèrent:] "Pourquoi tu casses les branches des arbres et tu [mets les bouteilles] dessus, c'est pour faire joli ?" Je leur ai dit que je respecte mes coutumes. Vous avez vidé la bouteille, mais les dieux eux aussi trouvent la vodka. Ils ont peut-être eu quelque part une bonbonne pleine. Ils doivent bien la verser quelque part. Je fais toujours comme ça. [On peut mettre des bouteilles] seulement au bout des branches de bouleau. Aux autres arbres on n'a pas le droit.

79 D'après Stepan, les dieux aspirent aux mêmes amusements que les humains. Et il semble que l'envie de vodka ou que les capacités d'absorption des dieux soient plus grandes que celles des hommes. À moins qu'ils n'aient le pouvoir surnaturel de trouver beaucoup de vodka à la fois. 
L'acte de mettre les bouteilles de vodka au bout des branches de bouleau confirme encore un lien avec le monde d'En-haut au cours du rituel. Traiter la vodka comme la boisson des divinités bienveillantes montre que l'alcool, en soi, spirituellement, n'est pas mauvais. C'est peut-être là un des éléments ambivalents dans la vision du monde des autochtones. La vodka peut vous mettre facilement sous l'effet des influences du monde d'En-bas, mais elle est aussi importante dans la communication avec les dieux du monde d'En-haut.

81 Nous voyons là aussi un élément didactique explicite pour la formation des jeunes Nenetses. Stepan n'a que rarement interféré publiquement au cours du rituel et, de manière générale, il n'a pas donné de leçons aux Nenetses sur la manière de mener leur rituel. Il a souligné à plusieurs reprises qu'il était invité, dans la mesure où il est khanty. Il a plutôt effectué des actes rituels qu'il considérait comme essentiels et que les Nenetses ne faisaient pas. En même temps, le comportement rituel des jeunes Nenetses le préoccupait dans la perspective de la durabilité du rituel. Il aurait préféré voir les jeunes maîtriser et appliquer de manière pertinente les savoir-faire rituels et les connaissances qui les accompagnent.

Stepan se plaignait de ce qu'aucun des jeunes n'ait eu la moindre idée de comment l'enchantement fonctionnait. À son avis, tout était fait de travers. On a mangé de la viande cuite avant que personne n'ait mangé de viande crue. D'après Stepan les gens auraient dû manger d'abord la viande crue, et seulement après la cuire. Stepan expliqua aux Nenetses dans quel ordre les choses devaient être faites, de sorte qu'il faut au moins manger un petit morceau de viande crue (là, toute la viande avait été bouillie). Une partie des gens mangea de la viande crue après. Au début, il n'y avait pas de sel, puis quelqu'un en apporta. Parmi les parties mangées crues, c'est avec un plaisir particulier que les gens dégustèrent le foie de renne, censé être le « snickers nenetse ». En même temps, tout le monde mangeait du renne bouilli.

\section{Suspendre les têtes et les peaux de renne au bouleau}

Quand ce deuxième repas fut achevé, les hommes prirent les trois peaux avec les têtes et les apportèrent près du bouleau qu'entre-temps Täkvjak avait choisi. Celui-ci, en quête du bon arbre, marcha en long et en large dans le bosquet et finit par montrer un arbre un peu plus grand que les autres. C'était l'un des rares où il était tout simplement envisageable de suspendre les peaux et les têtes.

Les hommes se dirigèrent vers l'arbre l'un après l'autre, à la queue leu leu. Un Nenetse grimpa à l'arbre. On lui lança le bout d'un lasso. L'homme fit passer le lasso par-dessus une branche et jeta en bas l'autre bout. Ainsi, à l'aide du lasso, les peaux et les têtes furent hissées l'une après l'autre. L'homme qui était au sommet de l'arbre les accrocha, en faisant passer les bois entre les branches. Ainsi les peaux restèrent suspendues, les têtes étant en haut.

Au moment où le premier renne fut hissé, les hommes crièrent ensemble sept fois. Ils entouraient l'arbre, les visages tournés vers celui-ci. Quand les trois rennes eurent été hissés sur l'arbre, les femmes commencèrent, à l'arrière, à crier elles aussi. Elles émirent à deux reprises un son hululant, puis restèrent silencieuses. Les hommes ne réagirent pas. Les femmes restèrent à l'arrière, les tournés visages vers l'ouest, mais c'était un hasard. Elles ne s'orientaient aucunement. 
Stepan expliqua ce qui s'était passé de la sorte, en actualisant une fois de plus les liens sacraux entre les mondes :

[Les peaux de rennes et les têtes ont été accrochées] sur le bouleau parce que le bouleau est un arbre sacré, on considère qu'il est en rapport avec le dieu blanc du ciel. Nous ne coupons jamais les bouleaux pour nous chauffer. C'est aux bouleaux qu'on les accroche. On offre aux dieux blancs un tissu blanc long de trois mètres ou un renne blanc. On l'accroche à l'arbre entièrement - avec la peau, la tête, les bois. Seulement en haut d'un bouleau [on l'accroche]. C'est un arbre sacré. Là de même on y accrochait des peaux. Seul le bouleau [convient]. Les autres, les pins ou les sapins, ne conviennent pas. Et les autres peuples, partout, vivent aussi de la sorte. Dans les autres régions.

La confirmation du lien et l'adresse aux dieux blancs ont donc eu lieu pendant la totalité du rituel, à toutes ses étapes. Stepan prêtait une grande attention à cette communication constante avec les divinités du ciel. Pour lui toute la cérémonie en était imprégnée, c'était l'action centrale, unifiant tous les détails du rituel.

\section{La fin du rituel}

Quand les hommes eurent accroché les peaux et les têtes à l'arbre, ils rangèrent et nettoyèrent tout ce qu'ils avaient apporté sur le lieu sacré - la viande, le reste des cendres, les lassos jetés dans les branches des bouleaux après le sacrifice - sur les motoneiges et sur les traîneaux et tous les participants partirent avec tout ce qu'ils avaient pour le village de Num-To.

89 Avant de partir tous durent faire sur place un petit tour dans le sens des aiguilles d'une montre. Tous les tournants, de manière générale, avec les traîneaux devaient être pris dans le sens des aiguilles d'une montre, aussi bien en partant qu'en arrivant. Un jeune Nenetse a fait un tour dans l'autre sens, ce que Stepan n'a pas manqué de remarquer. Le dernier à partir fut Täkvjak, le maître de la cérémonie, qui attendit que les autres fussent partis. En attendant, il chantait. Juri Vella est resté avec lui presque jusqu'au bout. Quand ils ne chantaient pas, ils étaient assis sur leur traîneau et ils souriaient.

91 La cérémonie était conclue. Le soir, après la cérémonie, les gens allèrent les uns chez les autres en visite au village de Num-To, pour boire du thé et chanter.

\section{Discussion : authenticité du rituel et politique}

92 L'interprétation de la cérémonie de sacrifice collectif tenue à Num-to en avril 1996 repose sur les explications de Stepan Randymov. Son action pendant la cérémonie m'a fait passer le message qu'il s'agissait d'une personne qui savait beaucoup sur le rituel et ses explications subséquentes ont conforté cette impression. Il s'est montré capable d'analyser les détails du rituel et en même temps d'en saisir l'ensemble et de le replacer dans un contexte religieux plus large.

93 La nature traditionnelle du rituel était dans une certaine mesure distincte de ses ambitions politiques. Pour Stepan, les efforts des militants pour les droits des peuples autochtones ne semblaient avoir aucune importance. En même temps, le message politique était clairement là et, pour ceux qui le faisaient passer, la justesse du rituel était en principe centrale. Les responsables de la dimension sacrale et de la dimension politique étaient différents et ils ne mélangeaient pas les genres. Les responsables du 
rituel permirent à toutes les personnes extérieures d'être présentes à la cérémonie et ils permirent que dans les intervalles de l'action rituelle il y ait des discours. Les représentants de la dimension politique laissèrent les responsables rituels faire tout ce qu'ils jugèrent bon. En même temps, on partait du principe qu'il ne fallait pas se tromper sur l'exigence d'authenticité et il y eut même quelques commentaires critiques à ce sujet (avec des doutes sur le sérieux avec lequel les gens de la région prenaient la justesse des détails du rituel).

La dimension politique s'est exprimée dans les discours tenus au début de la cérémonie, l'événement central étant les cadeaux de rennes faits au président de la Russie et au gouverneur de l'arrondissement de Hanty-Mansijsk. En même temps, les présents exprimèrent leur mécontentement face à l'extension de l'activité des compagnies pétrolières aux environs de Num-to. Le président et le gouverneur furent intégrés, d'après les coutumes nenetses, au même titre que les autochtones, comme victimes de la pollution de l'environnement en Sibérie occidentale.

Ce qui est aussi important, c'est qu'avant la cérémonie un appel avait été signé à Numto par les éleveurs de rennes, qu'ils présentèrent au gouverneur de l'arrondissement autonome. La déclaration exigeait que les terres limitrophes du lac soient préservées et soulignait l'importance de ne pas donner aux compagnies pétrolières des lieux sacrés.

En tant qu'action politique, la cérémonie publique de Num-to diffère des autres tentatives faites dans la région de faire fusionner des éléments de la culture traditionnelle des autochtones avec la lutte pour leurs droits. Jusque-là, cette lutte avait été composée de manifestations dont l'élément le plus original a été le čum (tente conique) dressé à des endroits critiques (sur la piste conduisant à un chantier pétrolier en 1990 et à Hanty-Mansijsk, face au bâtiment de l'administration en 1995) (Novikova 1999b ; 2000). La fusion d'un sacrifice et d'une protestation sociale était une démarche innovatrice, qui ne reposait sur aucun modèle, et cette entreprise dans son ensemble contenait un bon nombre d'éléments d'improvisation. C'était une tentative de lier la vision du monde et la pratique rituelle des autochtones avec les codes du langage politique dans une mesure nouvelle. Ce n'était pas facile d'atteindre l'équilibre, d'obtenir que l'authenticité du rituel soit assurée et que le message politique soit suffisamment fort et intelligible, y compris pour des hommes politiques et des fonctionnaires fort éloignés de la culture des autochtones.

La forme la plus forte d'expression du message rituel a été le cadeau fait par Juri Vella d'un renne au président de la Russie. Ce fait a été très largement commenté aussi bien dans la presse que dans des études scientifiques (Novikova 1998; 1999a ; 2002 ; Utrom 2003 ; Hanzerova 2009 ; Niglas, Toulouze 2004 ; Toulouze, Niglas 2012). Juri Vella n'a pas manqué de présenter publiquement par la suite la question du renne du président et l'a utilisée en tant qu'argument dans ses conflits ultérieurs avec LUKOIL (suivant la logique d'après laquelle l'arbitraire des travailleurs du pétrole met en danger le bien du président de la Russie, et menace le président en personne, du fait du lien sacral entre le renne et le président). C'est cet acte qui fait de la dimension politique de la cérémonie de Num-to un facteur durable. Ce rituel n'est pas resté un épisode exotique dans l'effort d'attirer l'attention sur les droits des peuples autochtones, mais sert de début à un discours politique imprégné de vision du monde autochtone, de fondement pour des initiatives ultérieures contre l'industrie pétrolière. Ce processus peut être compris en disant que si le rituel était authentique, la pratique politique anti-pétrole est aussi fondamentalement justifiée. 


\section{BIBLIOGRAPHIE}

$\mathrm{TT}=$ travaux de terrain de l'auteur.

BALzER Marjorie Mandelstam, 1999, The Tenacity of Ethnicity. A Siberian Saga in Global Perspective, Princeton University Press.

BARKAlajA Anzori, 1997, “On the Sacrificial Ritual of the Pim River Khanty in December 1995”, Pro Ethnologia 5, Tartu, p. 57-68.

BARKALAJA Anzori, 1999, “On the Sacrificial Rituals of the Pim River Khanties: Part Two”, Pro Ethnologia 8, Tartu, p. 57-72.

GEERTZ Clifford, 2001 [1973], The Interpretation of Cultures: Selected Essays, New York: Basic Books.

HANZEROVA 2009 = ХАНЗЕРОВА, ИРИНА, « ИСТОРИЯ ПРЕЗИДЕНТСКИХ ОЛЕНЕЙ », НАРЬЯНА ВЫНДЕР 01.08.2009.

KARJALAINEN Kustaa Frederik, 1918, Jugralaisten uskonto. Suomen suvun uskonnot III, Porvoo : Werner Söderström OY.

KULEMZIN 1980 = КУЛЕМЗИН В. М. « О ЧУВСТВЕ СТРАХА У ХАНТОВ-ОХОТНИКОВ », in ВОПРОСЫ ЭТНОКУЛЬТУРНОЙ ИСТОРИИ СИБИРИ, ТОМСК : ИЗДАТЕЛЬСТВО ТОМСКОГО УНИВЕРСИТЕТА, p. 107112.

KULEMZIN 1984 = КУЛЕМЗИН В. М., ЧЕЛОВЕК И ПРИРОДА В ВЕРОВАНИЯХ ХАНТОВ, ТОМСК : ИЗДАТЕЛЬСТВО ТОМСКОГО УНИВЕРСИТЕТА

LEETE Art, 1997, “Sacrificial Ceremony at Lake Num-To”, Pro Ethnologia 5, p. 35-55.

LEETE Art, 1999, "Ethnopolitical Comments About the Sacrificial Ceremony at the Lake Num-To", Pro Ethnologia 7, p. 21-26.

LEETE Art, Liivo NIGLAS, Anzori BARKALAJA, 2001, Põhjapõder arktilises kultuuris, Tartu : Eesti Rahva Muuseum.

LEETE Art, 2004, "Ritual, Politics and Mentality: The Nenets and Ob-Ugrians of Siberia", in AnnaLeena SIIKALA, Stein MATHISEN (eds.), Creating Diversities: Folklore, Religion and the Politics of Heritage, Studia Fennica Folkloristica 14, Helsinki: Finnish Literature Society, p. 125-135.

MOLDANOV, MOLDANOVA 2000 = МОЛДАНОВ, ТИМОФЕЙ, МОЛДАНОВА, ТАТЬЯНА, БОГИ ЗЕМЛИ КАЗЫМСКОЙ, ТОМСК : ИЗДАТЕЛЬСТВО ТОМСКОГО УНИВЕРСИТЕТА NIGLAS Liivo, 1997, "Reindeer in the Nenets Worldview", Pro Ethnologia 5, p. 7-33. NIGLAS Liivo, TOULouze Eva, 2004, "Yuri Vella's Worldview as a Tool for Survival: What Filming Reveals", Pro Ethnologia 17, p. 95-114.

NOVIKOVA 1998 = НОВИКОВА, Н.И., « КАК ЖИВЕТСЯ ОЛЕНЬЮ ПРЕЗИДЕНТА РОССИИ », ЖИВАЯ АРКТИКА, ИНФОРМАЦИОННЫЙ БЮЛЛЕТЕНЬ, о 1-2, С. 21-24.

NOVIKOVA 1999а = НОВИКОВА, Н.И., « ПРЕЗИДЕНТСКИЙ ОЛЕНЬ », ТРУД, 12. ФЕВРАЛЯ, 19. NOVIKOVA 1999b = НОВИКОВА, Н.И., « В ЧУМЕ ЖДУТ ОТВЕТА », НЕЗАВИСИМАЯ ГАЗЕТА 17.09.1999. 
NOVIKOVA 2000 = НОВИКОВА, Н.И., « ОТ ЧУМА НА ЦЕНТРАЛЬНОЙ ПЛОЩАДИ ДО СУДА » URL : http://jurivella.ru/vanaweb/index.php/2009-07-30-13-15-45/86-2009-08-20-16-43-50? format=pdf

NOVIKOVA 2002 = НОВИКОВА, Н.И., « КАК ЖИВЕТСЯ ОЛЕНЬЮ ПРЕЗИДЕНТА РОССИИ », in РАСЫ И НАРОДЫ, ВЫП. 28, ИНСТИТУТ ЭТНОЛОГИИ И АНТРОПОЛОГИИ ИМ. Н.Н. МИКЛУХО-МАКЛАЯ, МОСКВА : НАУКА, р. 133-146.

PROZES Jaak, 2013, „Inimene sünnib tõesti esivanemate hingest ! Juri Vella viimane intervjuu“, 10. märtsil 2013, Sirp 18.10.2013.

RADIN Paul 2008 [1956], “The Winnebago Trickster Figure”, in Michael LAMBEK (ed), A Reader in Anthropology of Religion, Blackwell Publishing, p. 226-237.

ROMBANDEEVA 1993 = РОМБАНДЕЕВА, Е.И., ИСТОРИЯ НАРОДА МАНСИ (ВОГУЛОВ) И ЕГО ДУХОВНАЯ КУЛЬТУРА (ПО ДАННЫМ ФОЛЬКЛОРА И ОБРЯДОВ), СУРГУТ : СЕВЕРНЫЙ ДОМ.

SCHMIDT Éva, 1989, “Bear Cult and Mythology of the Northern Ob-Ugrians”, in Mihály HOPPÁL (ed), Uralic Mythology and Folklore, Ethnologica uralica 1, Budapest: Ethnographic Institute of the Hungarian Academy of Sciences, p. 187-232.

SIIKALA Anna-Leena, 2000, "From Sacrificial Rituals into National Festivals: Post-Soviet Transformations of Udmurt Tradition", in Pertti J. Anttonen et al. (eds), Folklore, Heritage Politics and Ethnic Diversity. A Festschrift for Barbro Klein, Botkyrka, Sweden: Multicultural Centre, p. 57-85. TOULOUZE Eva, NIGLAS Liivo, 2012, “Native Spirituality in (Re)constructed Personhood: Observing and Filming Yuri Vella”, Folklore. Electronic Journal of Folklore, 51, Tartu, p. 133-170.

UTROM 2003 = « ЕЛЬЦЫН, ПУТИН И ПРЕЗИДЕНТСКИЙ ОЛЕНЬ », Freitag, 03.10.2003.

WIgET Andrew, BALAlaEVA Olga, 2011, Khanty, People of the Taiga: Surviving the $20^{\text {th }}$ Century, Fairbanks: University of Alaska Press.

\section{NOTES}

1. L'année précédente, Juri Vella avait organisé une manifestation à Hanty-Mansijsk en plantant une tente devant le bâtiment de l'administration de l'okroug autonome. Cette manifestation contre l'extension de l'industrie pétrolière avait soulevé quelques vagues (Novikova 2002). C'est pourquoi les compagnies pétrolières ne souhaitent pas de nouvelles manifestations.

2. Un million de roubles était équivalent en avril 1996 à environ 200 dollars.

3. Kaltaš-imi est la déesse principale des Khantys, qui donne à chaque personne, à la naissance, ses âmes et qui détermine aussi la longueur de la vie. Kaltaš est, dans les familles khantyes, l'esprit protecteur de la première fille. Elle est la mère ou la grandmère de la plupart des dieux, détermine le territoire où ils exercent leur autorité et leur confie des tâches particulières. Elle se présente sous forme d'un cygne. Les autres noms de Kaltaš-imi sont 'La Grande Femme qui a créé des Milliers de Déesses', 'La Grande Femme qui a créé des Milliers de Dieux', 'Cygne intouchable', 'la Mère [la plus âgée, la plus sage]', 'La Grande Femme aux cheveux d'Argent'. (Moldanov, Moldanova 2000, p. 37-50) 
4. La chance est une notion centrale dans l'univers mental autochtone: les rituels servent entre autres à en assurer la présence. C'est la chance qui donne des rennes en abondance, du gibier aux chasseurs, des poissons aux pêcheurs (NdT).

\section{RÉSUMÉS}

Dans cet article, j'analyse le sacrifice collectif de rennes organisé par les Khantys et les Nenetses des forêts en avril 1996 en Sibérie occidentale, au bord du lac Num-to, en utilisant la méthode de la description dense. J'étais présent à cette cérémonie, mais j'ajouterai à mes observations les explications données par les autochtones pour expliciter les conditions sémantiques, aussi bien de la tenue du rituel dans son ensemble que sur certains de ses éléments. Afin de montrer le contexte plus général, je m'intéresserai aussi aux liens entre ce rituel et le combat pour résister à la pression de l'industrie du pétrole. Cet article a ainsi pour objectif de montrer comment les Khantys et les Nenetses s'appuient sur leur vision du monde pour défendre leurs droits et comment la pratique rituelle permet d'intégrer ce combat à la vision du monde traditionnelle.

\section{INDEX}

Mots-clés : industrie du pétrole, lutte politique, place des femmes, religion autochtone, rennes, ritual, sacrifice, survie, vision du monde traditionnelle

Index géographique : Num-to, Neftejugansk, Belojarsk, Irtyš (rivière), Nižnevartovsk, Pim (rivière), Surgut, Tromagan (rivière), Khanty-Mansiïsk (Ougrie) (district autonome)

Thèmes : anthropologie, ethnologie, religion

nomsmotscles Khantys, Nénetses des forêts

disciplines russe

Keywords : gender issues, indigenous religion, oil industry, political struggle, reindeer, ritual, sacrifice, survival, traditional worldview, Forest Nenets, Khanty, beginning of the 21st century, Anthropology, Ethnology, Religion

Index chronologique : XXe siècle (début) 